\title{
Probabilistic Dispatch of Remote Hybrid Microgrids Including Battery Storage and Load Management
}

\author{
Claudia Battistelli, Member, IEEE, Yashodhan P. Agalgaonkar, Member IEEE, and Bikash C. Pal, Fellow, IEEE
}

\begin{abstract}
This work presents a probabilistic economic dispatch tool for energy management (EM) studies in the context of remote hybrid AC/DC microgrids (MGs). An EM approach is proposed to ensure a reliable power supply at the minimum cost of the hybrid MG operation. A comprehensive operational framework is presented, which considers topological features of the hybrid MG and the interlinking converter between $\mathrm{AC}$ and $\mathrm{DC}$ subsections. Approach and models are tested using several operating scenarios referred to a test hybrid MG system. In the analyses, the opportunity of integrating battery energy storage and energy demand management in the EM scheme is investigated. The results of the analyses demonstrate the effectiveness and practicality of the optimization tool in different operation contexts.
\end{abstract}

Index Terms - battery storage, economic dispatch, energy demand management, optimal operation, stand-alone hybrid microgrid

\section{NOMENCLATURE}

${ }_{t} \begin{aligned} & \text { Indices } \\ & t\end{aligned}$

Index for probabilistic scenarios, $s=1, \ldots, S$

Parameters

$B_{\text {cap }}$

$P_{B c h} \max$

$P_{B d c h} \max$

$P_{C G U} \max$

$P_{E V_{t}}^{0}$

$P_{I L C}$

$P_{L_{A C, t, s}}^{0}$

$P_{L_{D C, t, S}}^{0}$

$P_{L_{A C}^{c r i}, s}$

$P_{L_{D C}^{c r i}}^{c r s}$

$P_{P V-W P^{(d c)}} t, s$

Battery storage capacity

Maximum battery charge power

Maximum battery discharge power

Maximum power output of controllable generators

Scheduled electric vehicles' demand at $t$

Interlinking converter's rating

Scheduled demand from controllable AC load, at $t$ in $s$

Scheduled demand from controllable DC load, at $t$ in $s$

Scheduled demand from critical AC load, at $t$ in $s$

Scheduled demand from critical DC load, at $t$ in $s$

Combined solar-wind power generation in the DC sub-microgrid, at $t$ in $s$

This work was supported by the Engineering and Physical Sciences Research Council (EPSRC) UK through the "Reliable and Efficient Sys-tem for Community Energy Solution - RESCUES” grant. EP/K03619X/1: Data supporting this publication can be obtained on request from cap-publications@imperial.ac.uk.

$P_{W P^{(a c)} t, s}$
$R U_{C G U}$
$R D_{C G U}$
$S O C^{\min }$
$\Delta_{E V}^{\max }$
$\Delta_{L}^{\max }$
$\rho_{C G U}$
$\rho_{\Delta_{E V}}$
$\rho_{\Delta_{L}}$

Wind power generation in the AC sub-microgrid, at $t$ in $s$

Ramp up rate of controllable generators

Ramp down rate of controllable generators

Minimum state-of-charge of the battery

Maximum contracted reduction from scheduling for electric vehicles load

Maximum contracted reduction from scheduling for controllable loads

Unit cost of controllable generators' power production

Unit cost of electric vehicles' load curtailment

Variables

$P_{B_{t, s}}$

$P_{B c h_{t, s}}$

$P_{B d c h_{t, s}}$

$P_{C G U_{t, s}}$

$P_{E V_{t, s}}$

$P_{L_{A C, t, s}}$

$P_{L_{D C, t, s}}$

$P_{A C / D C_{t, s}}$

Unit cost of controllable loads' curtailment

Available battery storage capacity, at $t$ in $s$

Battery charge, at $t$ in $s$

Battery discharge, at $t$ in $s$

Controllable generators' power output, at $t$ in $s$

Final electric vehicles' load, at $t$ in $s$

Final AC controllable load, at $t$ in $s$

Final DC controllable load, at $t$ in $s$

Interlinking converter's power transfer from AC sub-microgrid to DC sub-microgrid, at $t$ in $s$

$P_{D C / A C t s} \quad$ Interlinking converter's power transfer from DC sub-microgrid to AC sub-microgrid, at $t$ in $s$

$\Delta_{E V_{t, s}} \quad$ Electric vehicles' load reduction at $t$ in $s$

$\Delta_{L_{A C, t S}} \quad$ Controllable AC load reduction at $t$ in $s$

$\Delta_{L_{D C, t, s}} \quad$ Controllable DC load reduction at $t$ in $s$

Binary variables

$X_{A C / D C_{t, s}} \quad 1$ for AC-to-DC power transfer at $t$ in $s, 0$ otherwise

C. Battistelli and B. C. Pal are with the Department of Electrical and Electronic Engineering, Imperial College, London, SW7 2AZ, UK (e-mail: c.battistelli@imperial.ac.uk; b.pal@imperial.ac.uk).

Y.P. Agalgaonkar was with the Department of Electrical and Electronic Engineering, Imperial College, London, SW7 2AZ, UK (e-mail: yashodhan.imperial@gmail.com). 


$$
\begin{aligned}
& X_{D C / A C_{t, s}} \\
& X_{c h_{t, s}} \\
& X_{d c h_{t, s}}
\end{aligned}
$$

and $\mathrm{AC}$ side through the interlinking converter. There has been some research effort in developing EM tools for hybrid MGs in [20], where an optimal power flow is presented for scheduling. However, the system configuration proposed in [20] cannot be associated with a typical MG model.

In light of the above, this paper proposes a methodological development in an EM framework for remote hybrid MGs installations, and in this respect its contribution is twofold. First, the paper systemically presents operation and management aspects that define realistic hybrid MG features and EM functionality. Second, it develops a comprehensive optimization-based approach, with related models, for the scheduling and dispatch of the MG's resources. The modeling considers the possible uncertain behavior of energy services.

Following the work in [17], [21] and [22], this paper proposes an EM scheme embedding battery energy storage and energy demand management for ensuring - even in uncertain conditions related to the fluctuations of certain parameters - a reliable power supply at the minimum operation cost of the hybrid MG. Then, the proposed optimization modeling approach is based on an economic dispatch (ED) problem formulation, where the objective is minimizing the costs of controllable generators and battery storage operation, as well as of energy demand management carried out on controllable loads, such as thermal loads or electric vehicles (EVs). Operating limits of controllable generators and interlinking converter, battery charge and discharge behavior, demand controllability limits, etc., are mathematically modeled and represented as constraints in the ED framework.

A realistic stand-alone hybrid MG is used to test effectiveness and potential of the EM approach. In the applications, the ED model is used to analyze the operating conditions of all hybrid MG resources that can be controlled and hence participate in the EM scheme, in a set of scenarios that can also correspond to different configurations of the hybrid MG structure. Particular focus is on the contributions of battery, energy demand management and interlinking converter.

The dispatch strategy depends on the hybrid MG configuration and composition. The sensitivity of such a strategy to any variation in the components of AC and/or DC sub-MGs can change case by case. Since the focus of this paper is on systemlevel power balance, the interlinking converter operation is mainly assessed through its power transfer potential between $\mathrm{AC}$ sub-MG and DC sub-MG, in relation to the behavior of the other resources.

In the presented problems, EM and operation of the hybrid MG are made robust to the uncertainties associated with loads and RESs. Accordingly, the ED model materializes in a probabilistic algorithm, where uncertain loads and RESs are stochastically modeled and all system components, functions and interactions are represented via the most proper mathematical formulation.

The simulations results demonstrate the practicality and robustness of both the EM framework and the ED tool, with respect to the operation of a stand-alone hybrid MG and the coordinated management of its resources.

The current proposal is the first result of a research work or DC MGs [16]-[19]. In hybrid MGs, the EM becomes more challenging because of the interconnection between DC side 
aimed at developing a comprehensive methodological platform for studies on electricity consumers gathered in isolated and possibly distributed MGs, tailored to the electrification needs of remote communities, and flexible to any type of DERs, RES' and loads, including battery storage systems and EV services. In this first work, the focus is on managing the active power dispatch for reliable load supply, whereas control aspects related to voltage and/or frequency stability, or to the behavior of the interlinking converter, are not addressed.

The rest of the paper is organized as follows. Section II describes in detail the EM methodology for a stand-alone hybrid MG. Section III presents the comprehensive mathematical developments of the ED model. A case study is presented in Section IV, along with simulation results and discussions. Finally, Section V concludes the paper.

\section{OPTIMIZATION-BASED ENERGY MANAGEMENT}

In a stand-alone hybrid MG with EM embedding battery storage and energy demand management, the EM system schedules the controllable generators in coordination with the operation of all other DERs, battery, and controllable loads, including the EVs. The objective is minimizing the overall operation costs of the hybrid MG while ensuring the continuity of power supply in relation to the end users' requirements. The EM approach depends on the hybrid MG framework, composition and configuration, and the optimized EM strategy is based on the assumption that security constraints on voltage and currents are ensured by the proper technological infrastructure (advanced metering, regulation and protection on lines and components.

The EM criteria is detailed in the following.

\section{A. Hybrid microgrid framework}

The basic reference MG is depicted in Fig. 1. The development of this system follows the paradigm of "smart user grid" [21], [22] where a complex MG can be seen as a grouping of several sub-MGs with suitable interfaces. In this framework, an AC sub-MG is connected to a DC sub-MG through an interlinking converter, so as to form a hybrid $\mathrm{MG}$ structure.

The AC sub-MG integrates a wind power plant (WP), controllable generating units (CGU), battery storage (BES), noncontrollable ("critical") AC loads (AC Lcri) and controllable AC loads (AC Lctrl). The DC sub-MG includes a photovoltaicwind system integrated with battery storage (PV-WP) [23], a unidirectional EV charging station, critical DC loads (DC Lcri) and controllable DC loads (DC Lctrl).

The PV-WP generation group in the DC sub-MG builds upon the system presented in [23], coupling a solar power unit and a wind power unit with similar capacity, and combining them with adequately sized battery storage to ensure a satisfactory continuity of power supply in relation to certain targets of power production. A proper control apparatus assists the coordinated functionality of the components.

In the basic reference MG configuration (Fig. 1), the EV station is equipped for unidirectional operation (grid-to-vehicle, $\mathrm{G} 2 \mathrm{~V}$, for charging only), and thus has no capability of backfeeding to the grid through power discharging. The reason of this choice is that, at present, the G2V technology is more viable

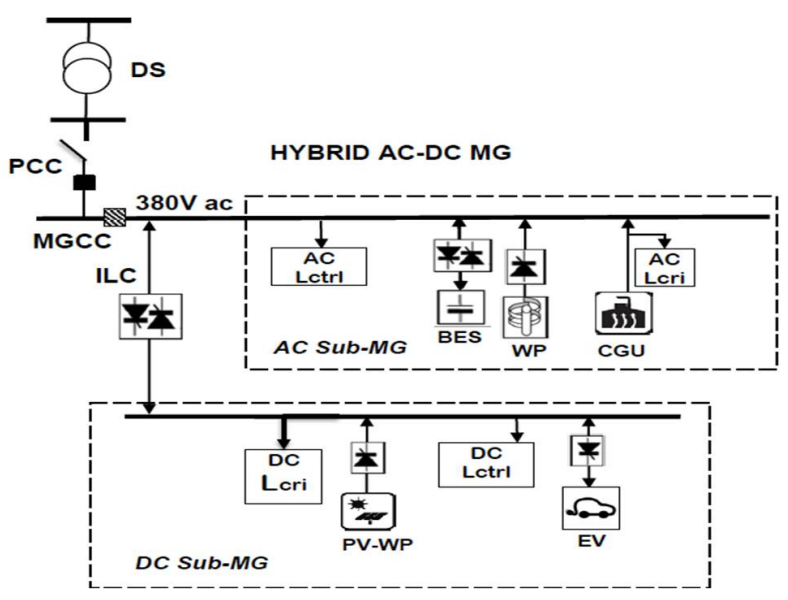

Fig. 1. Hybrid microgrid configuration

than vehicle-to-grid (V2G, bidirectional, for charging/discharging), due to the lack of relevant legislations and standards for the deployment and commercialization of the latter [24]-[26]. Nevertheless, it seems only a matter of time before V2G can happen, as there are future estimations and plans for it in various countries [24]-[26]. In view of this, it is interesting to evaluate the possible contribution of the $\mathrm{V} 2 \mathrm{G}$ service versus pure $\mathrm{G} 2 \mathrm{~V}$ operation, from the perspective of optimal EM. In this work, V2G is considered in an additional application of the proposed ED tool (ref. case G, Section IV), particularly to demonstrate the modeling flexibility within the considered study framework.

The hybrid MG is considered organized and operated as a multi-agent system, made of several domains (agents) of major functionalities: generation, delivery, storage, power conversion, monitoring, control, and interoperability [1].

\section{B. Energy management criteria}

Being stand-alone, the hybrid MG is reasonably considered under the jurisdiction of a local MG operator. This operator is the unique interface with the external environment and the actual aggregator of the MG resources, in charge for their operational planning and management related to technical factors (DERs' capabilities and operating limits) and economic aspects (costs, value, profits).

Individually non-dispatchable RESs, such as wind and solar generation, have to be totally used for energy saving and environmental benefit, leaving to the controllable generators a flexible supply provision according to rating and costs. Many studies have been carried out in the last decade, researching for possibly dispatchable solutions characterized by hybrid RESs combinations integrated with energy storage [23], [27], [28]. An example is the photovoltaic-wind power system considered in the hybrid MG under study, which however is not considered dispatchable.

The main duty of the battery storage (BES) is to compensate possible imbalances caused by non-controllable loads and generators, either supplying any power generation shortage or absorbing any extra-generated power. The battery is properly designed, and integrated in the AC sub-MG to ensure the best performance relative to MG-level load balancing support. In this 
respect, it is presupposed that the battery technology is suitable for long-duration service (e.g., lead-acid or sodium ion battery).

The energy demand management program entails the curtailment of the electricity demand of all controllable loads, including EV batteries. This program considers and achieves an equilibrium between the operating requirements of the hybrid $\mathrm{MG}$ and the impact on the energy customers. In the case of controllable loads such as thermal appliances, a certain temperature range cannot be violated, whereas for vehicles there are restrictions imposed by the desired state of charge of EV batteries for transportation purposes. For EVs, equipping the EV station for battery swapping service - which ensures the replacement of discharged batteries with fresh ones - can best suit the implementation of energy demand management [22]. In fact, the EV customers can contract the battery swapping service alongside the charging service, and the EV station operator can arrange a recharge-controlled battery stock as a local reserve, to be kept as an offer of lease to the EV customers if the energy demand management actions call for adjustments in their battery charging schedule.

In principle, $\mathrm{V} 2 \mathrm{G}$ service in MGs can address many tasks (e.g., RES generation smoothing, reactive power/voltage control, power quality, peak load leveling, etc.), but the technical and economic feasibility of any of them depends on various factors related to system configuration, control, operation program, and market strategy of both distribution network and EV aggregator [29]. A systemic analysis of V2G realization and employment is beyond the scope of this paper and deferred to future studies. As detailed later, in this work, V2G is included in the rationale of the EM program as a support to load leveling, enhancing the load management performance and effectiveness.

\section{Objectives of optimization-based energy management}

The EM goal is optimizing the following actions: a) Scheduling of the controllable generators' power output.

b) Curtailment of all controllable loads, including EV recharging, based on the energy demand management criteria.

c) Scheduling of battery energy storage charge/discharge.

d) Power exchange between AC sub-MG and DC sub-MGs through the interlinking converter.

The power balance is primarily achieved by the generating resources. Energy demand management is applied to cover the remaining power balance requirements.

\section{ECONOMIC DISPATCH}

The presented methodology involves a preliminary network analysis for the hybrid MG, aimed at verifying and calibrating the ED constraints in the configuration and all the operating conditions considered for the optimization studies.

The following modeling components refer to the hybrid MG structure of Fig. 1. Since the focus is on economic dispatch of active power, all converters (DERs' interfaces and interlinking converter) are assumed working at unity power factor, so the effects of reactive power are not represented. a) Local DERs - wind power unit in the AC sub-MG, and battery-integrated photovoltaic-wind system in the DC sub-MG

The electricity generated by the wind power unit in the AC sub-MG is related to the wind speed profile [30]-[31] whereas the photovoltaic-wind generation system with battery storage in the DC sub-MG can supply self-produced power independently of solar radiation conditions [23], [28]. In order to account for the uncertainties in the power produced by these DERs, Monte Carlo Simulation is employed to generate a large number of daily scenarios, starting from realistic 24-hours power output profiles with variations probabilistically modeled through a normal distribution. The sampling is done by assuming the consecutive (hourly) operating states independent.

Scenario Reduction [32] is used to eliminate the scenarios with lowest probability and aggregate close scenarios, though respecting four basic criteria, namely: 1) the "reduced" set of scenarios must be good enough to characterize the stochastic variables; 2) it must capture all events and their likelihood; 3) it must be as small as possible to avoid computational intractability; 4) the resulting solution of the stochastic problem must indicate that the addition of more scenarios to the optimization does not change significantly the optimal value of the objective function.

A normal distribution is the simplest assumption about the interdependence structure of the random variables, and it is probably the best model when there are no particular contextual conditions to characterize the possible deviations from the normal distribution itself [30]-[31], [33]. In the most usual cases, other statistical distributions - considering slight deviations (e.g., Rayleigh, Weibull) - can be similarly considered [31].

\section{b) Non-controllable and controllable loads}

Hourly (24-hours) probabilistic scenarios are modeled referring to typical profiles of residential and commercial loads, with deviations based on a normal probability distribution function [34].

Monte Carlo Simulation and Scenario Reduction technique are applied for the modeling, in the same way as for the DERs.

\section{c) Operating constraints of the controllable generating units}

The constraints in controllable generators' power production are as follows:

$$
\begin{aligned}
& 0 \leq P_{C G U_{t, s}} \leq P_{C G U} \max \\
& P_{C G U_{(t+1), s}}-P_{C G U_{t, s}} \leq R U_{C G U} \\
& P_{C G U_{t, s}}-P_{C G U_{(t+1), s}} \leq R D_{C G U}
\end{aligned}
$$

Constraints (1) enforce the controllable generators' power supply limits, whereas constraints (2) and (3) control the ramp down/up rates. Operating limits and rates depend on the electrical characteristics of the generators.

\section{d) Operating constraints of the battery energy storage}

The battery operation is constrained by the limits in storage capacity and charge/discharge rates, as follows:

$$
\begin{aligned}
& 0 \leq P_{B c h_{t, s}} \leq X_{c h_{t, s}} \cdot P_{B c h}^{\max } \\
& 0 \leq P_{B d c h_{t, s}} \leq X_{d c h_{t, s}} \cdot P_{B d c h} \max
\end{aligned}
$$


$X_{c h_{t, s}}+X_{d c h_{t, s}} \leq 1$

$P_{B_{t, s}} \leq B_{\text {cap }}$

Constraints (4) and (5) limit the battery charge/discharge, with $X_{c h_{t, s}}$ and $X_{d c h_{t s}}$ being the " $0 / 1$ " binary variables defined in (6) to account for the battery charge/discharge cycles. Constraints (7) enforce the limits for the available battery capacity. The hourly available battery capacity is defined by:

$P_{B(t+1), s}=P_{B t, s}+\frac{P_{B c h_{t, s}}+P_{B d c h_{t, s}}}{B_{c a p}}$

Model (4)-(8) strictly relates to the electrical characteristics of the energy storage, and does not depend on the electrical configuration of the MG.

\section{e) Interlinking converter}

In an ED problem, the simplest and yet most appropriate approach to model the interlink converter is by its AC-to-DC and DC-to-AC power conversions [35]-[36]. The role of the device is critical to maintain power balance at a MG level with an adequate power transfer between $\mathrm{AC}$ sub-MG and DC sub-MG, which mainly depends on conversion's rated capacity and efficiency [7].

In this work, the control is assumed as intrinsically addressed and solved a-priori, through an appropriate technological design and selection. Accordingly, the control aspects of the power conversion are not considered in the modeling, which is specifically oriented to the dispatch problem. Hence, the operating constraints can be formulated as follows:

$0 \leq P_{A C / D C_{t, s}} \leq X_{A C / D C_{t, s}} \cdot P_{I L C}$

$0 \leq P_{D C / A C_{t, s}} \leq X_{D C / A C_{t, s}} \cdot P_{I L C}$

$X_{A C / D C_{t, s}}+X_{D C / A C_{t, s}} \leq 1$

Inequalities (9) and (10) enforce the limits for the power conversion between AC side and DC side. Inequality (11) represents the " $0 / 1$ " binary variables $X_{A C / D C_{t, s}}$ and $X_{D C / A C_{t, s}}$ to account for the direction (either AC-DC or DC-AC) of the power converted.

\section{f) Energy demand management}

The energy demand management criteria on controllable loads and EVs can be represented by proper "contracted bands" of possible reduction of the scheduled demand. These bands are set up by agreement between the hybrid MG operator and the electricity customers [22].

\section{- Energy demand management on controllable loads}

Controllable loads are grouped into AC and DC load aggregations featuring some inherent physical properties, the most important of which are aggregated thermal capacity, number of load units and aggregated rated power. The contracted bands of load reduction are mainly constrained by the aggregated rated power and the customers' requirements [22], [37]. Accordingly, the contracted bands for the curtailment of the controllable loads are formulated as follows:

$0 \leq \Delta_{L_{A C, t, S}} \leq \Delta_{L}^{\max }$
$0 \leq \Delta_{L_{D C, t, S}} \leq \Delta_{L}^{\max }$

Constraints (12) and (13) ensure that the curtailed amount of controllable loads stays within the contracted limits at all times.

\section{- Energy demand management in the EV station}

EVs participation in energy services' provision is still at a conceptual stage, and neither recognized rules nor references exist to assign contracted bands to their management. Even for EV load management aimed at the economic dispatch of a hybrid MG - which is the case focused in this work -, different criteria can be used, based on the scope and framework of study [22].

In general, the contracted bands of curtailment for the EV charging service can be reasonably assumed dependent on technical factors (state of charge and maximum charge/discharge of the EV batteries) and on the type of agreement established with the EV customers. The latter must prioritize customers' convenience at every time. Based on this, the contracted bands for the EV load reduction are formulated as follows [22]:

$0 \leq \Delta_{E V_{t, s}} \leq \Delta_{E V} \max$

Constraints (14) limit the amount of EVs charging demand that can be reduced over the hours of the scenarios considered.

In case of EV service management in V2G mode, the connection of the EV station to the DC bus leads to a unidirectional interaction with the DC bus, and to a unidirectional effect on the whole system via the DC bus. For this reason, as later shown in Section IV, 'Case G' refers to a V2G dispatch for DC bus load leveling, as an ancillary service to the hybrid MG economic operation. The V2G service is considered as a scheduled resource, assuming that the EV station operator can set the EV charge/discharge program in "quasi real-time", based on metering apparatus and bidirectional communication network distributed in the MG [21]. This is nowadays realistic, considering the advancement of technology, and given the possibility to include battery swapping in the EV station, which allows the EV operator to reserve a specified EV power potential for the grid support [38].

Either in G2V or in V2G mode, constraints (14) consider the condition that all batteries admitted to the discharging schedule comply with prefixed limits for the state-of-charge.

\section{g) Cost of battery operation for power balance}

The battery is operated to exchange power with the hybrid MG by charge/discharge, based on the generation/load balance needs. The cost of this service is dictated by the amount, the specifications, the reliability, the time, and the frequency of charge/discharge cycles contracted by the MG operator [39], but can be aggravated by existing organizational and operational constraints, as well as financial obligations (debts, loans, etc.) for the battery owner. Possible uncertainties and corrective factors can also influence the cost, in relation to the jurisdiction, the organization and the operation of the MG. All this is considered in the proposed EM system, and represented in the ED tool by a cost function associated to the battery cycling. 
The cost function proposed in this paper builds upon an accredited battery cost model available in [40], correlating the hourly cost of battery operation $\left(\operatorname{Cost}_{B_{t, s}}\right)$ to a base cost coefficient $\left(c_{B}\right)$, state-of-charge and hourly available battery capacity. Its formulation is as follows:

$$
\operatorname{Cost}_{B_{t, s}}=\left[c_{B} \cdot k_{S O C_{\text {min }}}\left(1-\operatorname{SOC}^{\text {min }}\right)\right] \cdot P_{B_{t, s}}
$$

Compared to the model in [40], function (15) is more accurate in the definition of $c_{B}$. In fact, while $c_{B}$ is simplified in [40] as a mere cost of battery wear, obtainable from the manufacturing information, this paper defines the cost coefficient - in principle - as a function of all the aforementioned factors. In practice, the value of $c_{B}$ is determined by the factors - among those mentioned - that are actually relevant and specific to the case study.

In (15), $k_{S O C_{\min }}$ is a scalar parameter associated to the minimum state of charge of the battery. It can be derived by the manufacturing information.

Equation (15) implies that it is more economical to operate a battery at a higher state of charge. Physically, this is because low state of charge causes mechanical stress on the active masses and battery sulfation in lead-acid batteries.

\section{h) Complete economic dispatch model}

Considering the stochastic modeling of RESs' and loads' behavior, the complete ED model materializes into a probabilistic mixed integer nonlinear program (MINLP), the objective of which is determining the minimum cost of operating the controllable hybrid MG's resources, subject to a certain number of constraints, as follows.

\section{- Objective function}

$\operatorname{Min} E\left(\operatorname{COST}_{H M G}\right)=f\left(P_{C G U_{t, s}}, P_{B_{t, s}}, \Delta_{E V_{t, s}}, \Delta_{L_{A C, t, s}}, \Delta_{L_{D C, t, s}}\right)=$ $\sum_{s} \pi(s) \sum_{t}\left[\operatorname{Cost}_{C G U_{t, s}}+\operatorname{Cost}_{B_{t, s}}+\operatorname{Cost}_{\Delta_{E V_{t, s}}}+\right.$ $\left.\operatorname{Cost}_{\Delta_{L_{A C, t, s}}}+\operatorname{Cost}_{\Delta_{L_{D C, t, s}}}\right]=\sum_{s} \pi(s) \sum_{t}\left[\left(\rho_{C G U} \cdot P_{C G U_{t, s}}\right)+\right.$ $\left.\operatorname{Cost}_{B_{t, s}}+\left(\rho_{\Delta_{E V}} \cdot \Delta_{E V_{t, s}}\right)+\left(\rho_{\Delta_{L}} \cdot \Delta_{L_{A C, t, s}}\right)+\left(\rho_{\Delta_{L}} \cdot \Delta_{L_{D C, t, s}}\right)\right]$

This represents the expected costs of operating the hybrid MG. It is calculated as the summation over time $t$ (where $t$ is a hour), in all weighted probabilistic scenarios $s(\pi(s)$ : probability/weight of scenarios), of the following terms:

a) $\operatorname{Cost}_{C G U_{t, s}}=\rho_{C G U} \cdot P_{C G U_{t, s}}$ : cost of power supply from controllable generators at $t$ in $s\left(P_{C G U_{t s}}\right.$ : controllable generators' power output at time $t$ in scenario $s, \rho_{C G U}$ : unit cost of controllable generators' power production);

b) $\operatorname{Cost}_{B E S_{t, s}}$ : battery cost at $t$ in $\mathrm{s}$, defined by function (15);

c) $\operatorname{Cost}_{\Delta_{E V} t, s}=\rho_{\Delta_{E V}} \cdot \Delta_{E V_{t, s}}$ : cost of EV load curtailment at $t$ in $s$;

d) $\operatorname{Cost}_{L_{A C}, s}=\rho_{\Delta_{L}} \cdot \Delta_{L_{A C, t, s}}$ : cost of controllable AC load curtailment at $t$ in $s$;

e) $\operatorname{Cost}_{L_{D C}, s}=\rho_{\Delta_{L}} \cdot \Delta_{L_{D C, t, s}}$ : cost of controllable DC load curtailment at $t$ in $s$.
In the objective function, all cost terms are minimized simultaneously. "Importance" weights $\left(0<w_{i}<1\right)$ could be integrated as coefficients of the individual costs in order to minimize these costs with priorities. Moreover, each probabilistic scenarios is assigned probability/weight $\pi(s)$ equal to 1 , under the assumption of scenarios $s$ having the same realization likelihood.

- Constraints (1) - (14)

These have been discussed in the previous sub-sections.

- $\quad \underline{\mathrm{AC}}$ and DC sub-MG power balance equations

$P_{C G U_{t, s}}+P_{W P^{(a c)} t, s}+P_{B d c h_{t, s}}+P_{D C / A C_{t, s}}=P_{L_{A C, t, s}}+$

$P_{L_{A C} c r i}+P_{B c h_{t, s}}+P_{\frac{A C}{D C} t, s} \quad \forall t, s$

$P_{P V-W P^{(d c)}}{ }_{t, s}+P_{A C / D C_{t, s}}=P_{L_{D C, t, s}}+P_{L_{D C}^{c r i}, s}+P_{E V_{t, s}}+$

$P_{D C / A C_{t, s}} \quad \forall t, s$

Equations (16) and (17) ensure the power balance at $t$ in $s$, respectively in AC sub-MG and DC sub-MG.

- Definition of controllable loads' and EVs charging demand

$P_{L_{A C, t, s}}=P_{L_{A C, t, s}}^{0}-\Delta_{L_{A C, t, s}} \quad \forall t, s$

$P_{L_{D C, t, s}}=P_{L_{D C, t, s}}^{0}-\Delta_{L_{D C, t, s}} \forall t, s$

$P_{E V_{t, s}}=P_{E V_{t}}^{0}-\Delta_{E V_{t, s}} \quad \forall t, s$

Equations (18) - (20) define the load reductions $(\Delta)$ operated by the MG operator - based on the energy demand management and the power balance needs - and the corresponding final loads, for: AC controllable loads (equation (18)), DC controllable loads (equation (19)), and EVs (equation (20)).

The above ED problem is formulated neglecting power losses. This is possible in the case of small MG installations for remote communities (such as the proposed case study), where the system losses are typically not so significant given the short distances between components [41].

\section{CASE STUdY}

The results of applying the ED model to the test stand-alone hybrid MG of Fig. 1 are presented in this section. All analyses are performed for $t=1, \ldots, 24$ hours, using KNITRO solver engine. The following cases are considered:

- Case A: base case used to validate the ED tool.

- Case B: loss of a controllable generating unit.

- Case C: energy storage is not part of the EM system.

- Case D: the interlinking converter capacity is progressively reduced up to the minimum possible, at unity power factor.

- Case E: the EVs have reduced controllability due to exclusion of swapping service.

- Case F: the EM is influenced by the mechanisms of electricity markets and the possibly varying ratio of renewable and controllable resources to non-controllable ("critical") demand.

- Case G: the possibility of V2G contribution to the EM of the hybrid MG is considered, in the same framework and conditions as Case F's.

The abovementioned cases are specifically selected to evaluate and highlight the practicality and flexibility of use of EM 
approach and models. Following Case A, Cases B-E focus specific operation and management aspects, demonstrating that the ED tool is effective to assess the operating status of the hybrid MG in different scenarios and problems. Particularly, these cases address technical aspects, such as reliability (e.g., Cases $\mathrm{B}$ and $\mathrm{C}$ ), load imbalance (Case E), or the advantages of EM systems with battery energy storage (Case $\mathrm{C}$ ) and/or energy demand management (Cases $\mathrm{C}$ and $\mathrm{E}$ ). Case F, instead, is particularly market-oriented, assessing the $\mathrm{MG}$ operation in one of the typical time-based electricity pricing frameworks. Finally, Case $\mathrm{G}$ assumes that the EV charging station is equipped with a storage stock obtained by onboard/onsite EV batteries, of sufficient capacity to provide V2G ancillary service, based on a strategy to address load leveling at the DC bus through the optimization tool. This strategy can improve the total power absorption profile of the hybrid MG and thus its energy efficiency.

With respect to all considered cases, the topological features of a stand-alone hybrid MG have to be critically evaluated in relation to different scenarios and problems, in order to make the MG's operational framework robust against contingencies. This has been done for the hybrid MG under study.

The quantitative and qualitative results of the assessments represent a database that the hybrid MG operator can use for planning ahead actions on the system, related not only to the operation, but also to contingency management and maintenance.

\section{A. Assumptions and data in Case A}

The base case considers the availability of all hybrid MG's

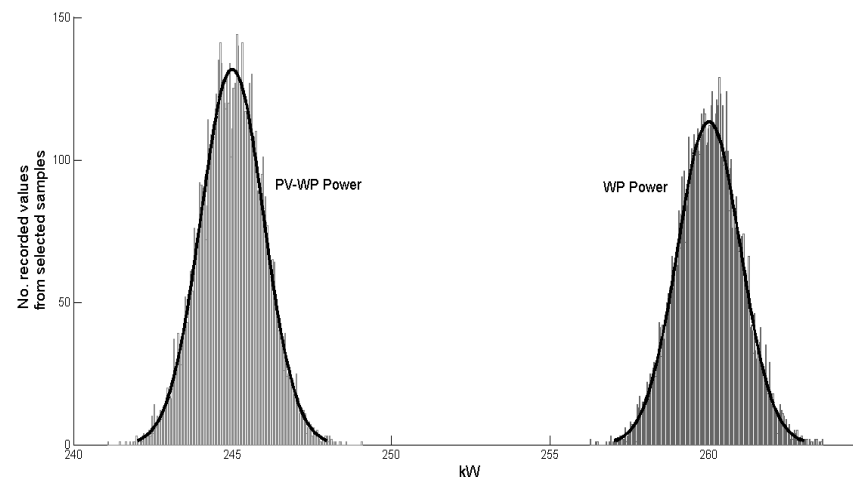

Fig. 2. Probabilistic distribution of wind and combined solar-wind power at a reference hour of the day (12 p.m.)

resources, with data modeled in accordance to the typical LV power distribution levels $(\mathrm{kW})$.

- Controllable generation: the grouping of two $250 \mathrm{~kW}$ units is considered. Data are given in Table I.

- RESs: Referring to the data in [22] for the wind power unit, and to the data in [23] and [28], properly rescaled, for the battery-integrated photovoltaic-wind power system, the set of probabilistic scenarios of power outputs for these DERs is obtained through combined Monte Carlo Simulation and Scenario Reduction technique, following the criteria described in paragraph a) of Section III. A set of 10,000 hourly (24-hours) scenarios, normally distributed, is generated by Monte Carlo Simulation, and this set is reduced to 1,000 samples by Scenario

\begin{tabular}{|c|c|c|c|c|}
\hline \multicolumn{5}{|c|}{$\begin{array}{c}\text { TABLE I } \\
\text { CONTROLLABLE GENERATION AND BATTERY BANK DATA }\end{array}$} \\
\hline $\begin{array}{c}P_{\text {CGU }}^{\max } \\
{[\mathrm{kW}]}\end{array}$ & $\begin{array}{c}\boldsymbol{R} \boldsymbol{U}_{\boldsymbol{C G U}} / \\
\boldsymbol{R D}_{\boldsymbol{C G U}} \\
{[\mathrm{kW} / \mathrm{h}]}\end{array}$ & $\begin{array}{c}\boldsymbol{B}_{\text {cap }} \\
{[\mathrm{kWh}]}\end{array}$ & $\begin{array}{c}P_{B c h}^{\max } / \\
P_{B d c h}^{\max } \\
{[\mathrm{kW}]}\end{array}$ & $S O C^{\min }$ \\
\hline 500 & 200 & 40 & 35 & 0.20 \\
\hline
\end{tabular}

\begin{tabular}{|c|c|c|c|c|c|c|}
\hline \multicolumn{7}{|c|}{$\begin{array}{c}\text { TABLE II } \\
\text { WIND POWER, SOLAR POWER, CRITICAL LOADS AND ENER }\end{array}$} \\
\hline Hour & Mean $P_{W P^{(a c)}} t^{[\mathbf{k W}]}$ & Mean $P_{P V-W P^{(d c)}} t[\mathbf{k W}]$ & Mean $P_{L_{A C_{t}}^{c r i}}[\mathrm{~kW}]$ & Mean $P_{L_{D C_{t}}^{c r i}}[\mathrm{~kW}]$ & $\rho_{\Delta_{E V, t}}[\boldsymbol{€} / \mathbf{k W h}]$ & $\rho_{\Delta_{L, t}}[€ / \mathbf{k W h}]$ \\
\hline 1 & 253.24 & 268.32 & 171.5 & 180.94 & 0.122 & 0.157 \\
\hline 2 & 261.59 & 238.17 & 164.55 & 182.27 & 0.122 & 0.178 \\
\hline 3 & 256.87 & 222.64 & 164.26 & 180.1 & 0.122 & 0.161 \\
\hline 4 & 257.92 & 264.05 & 168.21 & 181.77 & 0.138 & 0.154 \\
\hline 5 & 254.01 & 238.25 & 165.23 & 192.81 & 0.185 & 0.152 \\
\hline 6 & 260.2 & 239.01 & 166.46 & 174.41 & 0.174 & 0.153 \\
\hline 7 & 261.71 & 230.07 & 176.11 & 189.31 & 0.236 & 0.155 \\
\hline 8 & 263.07 & 225.59 & 169.58 & 175.23 & 0.265 & 0.158 \\
\hline 9 & 261.88 & 222.57 & 174.3 & 174.6 & 0.276 & 0.153 \\
\hline 10 & 265.25 & 246.26 & 164.84 & 176.44 & 0.274 & 0.16 \\
\hline 11 & 256.42 & 227.22 & 166.19 & 178.02 & 0.263 & 0.153 \\
\hline 12 & 260.53 & 245.15 & 166.23 & 175.64 & 0.253 & 0.154 \\
\hline 13 & 263.5 & 230.6 & 182.72 & 186.41 & 0.25 & 0.167 \\
\hline 14 & 264.59 & 240.31 & 162.66 & 184.42 & 0.261 & 0.163 \\
\hline 15 & 255.96 & 255.13 & 165.91 & 177.96 & 0.263 & 0.161 \\
\hline 16 & 257.07 & 237.54 & 170.57 & 179.71 & 0.26 & 0.161 \\
\hline 17 & 255.54 & 220.57 & 178.42 & 183.26 & 0.26 & 0.157 \\
\hline 18 & 262.93 & 227.18 & 171.99 & 193.55 & 0.276 & 0.153 \\
\hline 19 & 261.55 & 226.15 & 184.87 & 174.64 & 0.236 & 0.161 \\
\hline 20 & 265.23 & 247.32 & 165.66 & 179.02 & 0.174 & 0.164 \\
\hline 21 & 262.89 & 232.11 & 177.66 & 179 & 0.141 & 0.166 \\
\hline 22 & 256.24 & 236.22 & 165.73 & 174.14 & 0.133 & 0.17 \\
\hline 23 & 263.28 & 234.18 & 169.22 & 191.73 & 0.133 & 0.172 \\
\hline 24 & 266.48 & 239.76 & 164.81 & 180.64 & 0.133 & 0.155 \\
\hline
\end{tabular}


Reduction. In the Monte Carlo Simulation, the standard deviation is assigned as constant over the 24 hours, assuming that it is not significantly influenced by variations during the day. The hourly mean values (over all probabilistic scenarios) of the power output profiles are given in Table II, whereas Fig. 2 shows the probabilistic distribution of the data dissemination diagrammed for the RESs outputs, at one particular hour of the day (12 p.m.).

- Battery storage: a lead-acid battery bank, suited to long-term use, is considered. The technical data are provided in Table I.

- Non-controllable and controllable loads: $s=1, \ldots, 1000$ different scenarios of demand are derived via combined Monte Carlo Simulation and Scenario Reduction techniques, referring to typical residential and commercial aggregated profiles. Controllable loads represent aggregations of thermal loads. Hourly mean values (over all probabilistic scenarios) of both AC and DC loads are given in Table II.

- EV service: this is equipped for both EV battery recharging and EV battery swapping services. Two charging platforms are considered: $400 \mathrm{~V} / 32 \mathrm{~A}-24 \mathrm{~kW}-3$ phase for normal (2 h) charging, and $400 \mathrm{~V} / 63 \mathrm{~A}-43 \mathrm{~kW}-3$ phase for fast (30 min) charging. The number of batteries available for swapping is assumed enough to cover both G2V car fleet and V2G units, also considering load curtailment if needed. The EV charge scheduling is modeled considering normal and fast charging, based on a preestablished energy demand management program. The 24hours values of these profiles are displayed in the "EV load" subplots of Figs. 3 and 4 (ref. "initial" profiles), and in Fig. 7.

- Interlinking converter: it is formed by a bank of three $500 \mathrm{kVA}$ bidirectional converters, all with $100 \%$ conversion efficiency in both directions (AC-DC and DC-AC), for a total base rating of 1.5MVA.

- Costs: the unit cost of controllable generators' power is considered fixed. The reference value $\left(\rho_{C G U}=0.06 € / \mathrm{kWh}\right)$ is taken from historical pricing data. Costs of energy demand management on controllable loads and EVs are referred to a dynamic pricing structure. These costs are slightly higher than controllable generators', related to the resources' size and features [42]. The battery cost coefficient $c_{B W}$ is assumed equal to $0.107 € / \mathrm{kWh}$, and scalar parameter $k_{S O C_{\min }}$ is assumed equal to 0.15 .

- Contracted bands of energy demand management on controllable loads and EVs: they are hierarchically assigned based on the expected demand requirements: $75 \%$ of the scheduling is assumed for controllable loads, and $85 \%$ of the scheduling for EVs. The higher value of the EV contracted band complies with the structure and organization of the EV station, which is provided with swapping facilities in case the charging cannot be accomplished.

\section{B. Results \\ a) Case A}

The results for Case A are depicted in Fig. 3, which shows the hourly mean values (over all probabilistic scenarios) of the operating conditions of the hybrid MG resources, in terms of: active power dispatch of the controllable generators; "available battery capacity" (i.e., the remaining storage space in the battery bank, consequent to the number of charge/discharge cycles in a hour); interlinking converter's power transfer from AC subMG to DC sub-MG; curtailed (final) profiles, vs. initial (scheduled) demand, of - respectively - controllable AC loads, controllable DC loads and EVs.

From Fig. 3, "CGU power dispatch" subplot, it can be observed that the controllable generation works at almost full capacity during daytime, supplying less electricity during night and early morning. This outcome is reasonable considering the lower controllable generation's cost. Also, from the related subplots, it can be seen that the EV load is subject to some curtailment only during night-time, whereas the controllable AC and DC loads are curtailed during the daytime. This is due to the costing dynamics of the energy demand management, for which the controllable loads are cheaper during most of the times (ref. unit costs in Table II). The "available BES capacity" subplot shows the battery being almost emptied during night and early morning times (in fact, the available storage space almost
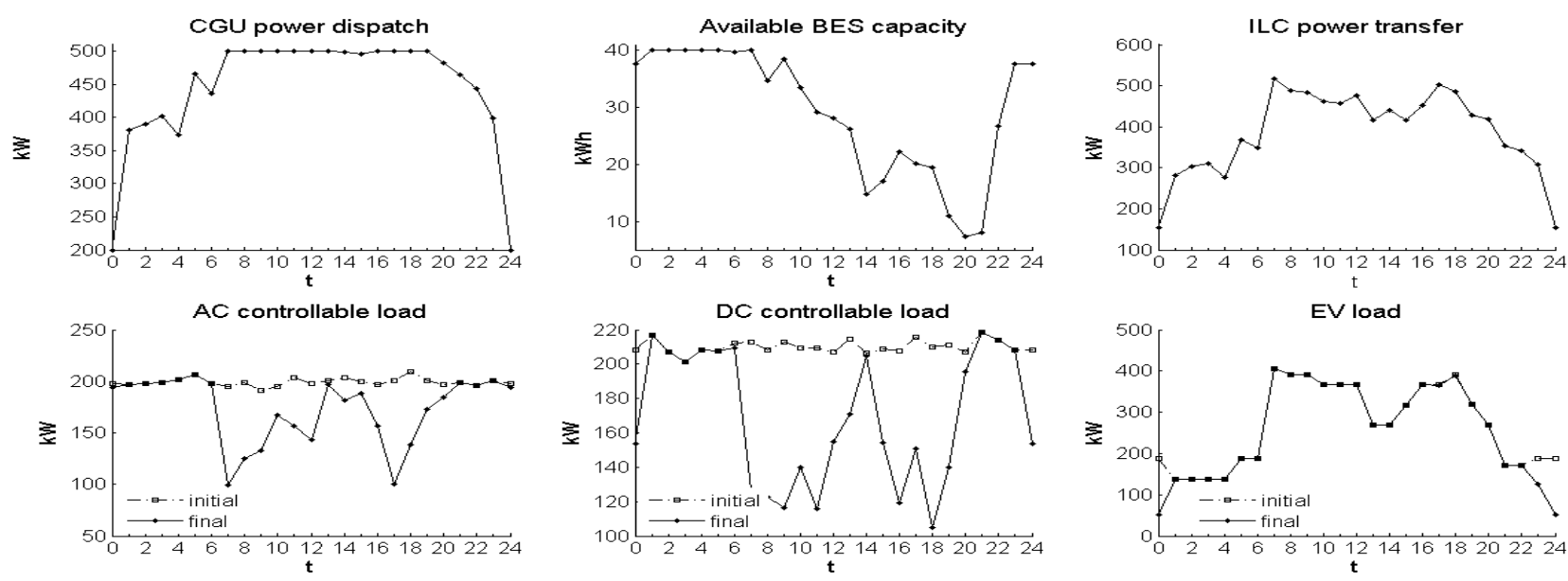

Fig. 3. Optimal operating conditions of the hybrid MG resources in Case A 

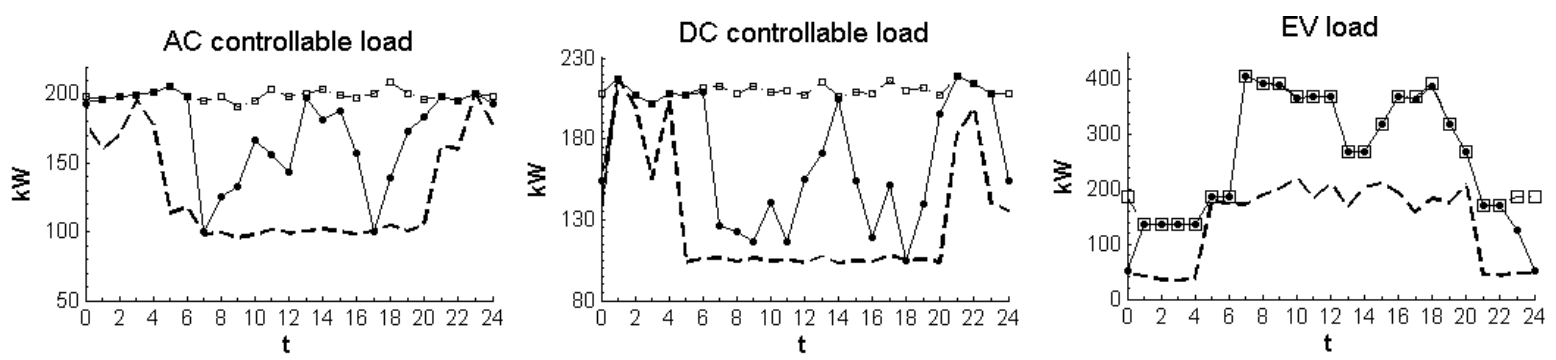

$-\cdot \cdot \cdot$ Initial (scheduled) $\longrightarrow$ Final - Case A - - Final - Case B

Fig. 4. Operating conditions of controllable loads following the loss of controllable generation capacity (Case B)
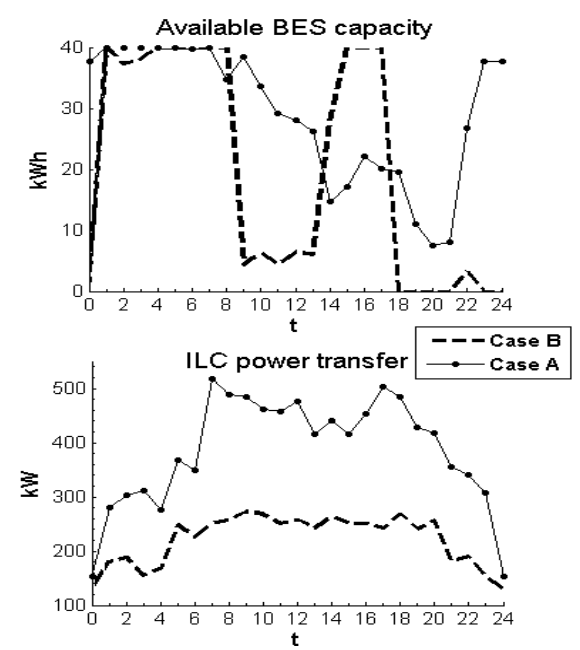

Fig. 5. Operating conditions of interlinking converter and battery storage following the loss of controllable generation capacity (Case B)

equals the maximum capacity), which means that when the hybrid MG cannot count either on electricity supply from the controllable generation, or on load curtailment, the load balance requirements are covered by the battery alongside the RESs. This outcome demonstrates the effective coordination and interoperability of the MG resources based on the operated EM program.

With respect to the interlinking converters' power transfer, which is shown in the "ILC power transfer" subplot of Fig. 3, it is directed from the AC sub-MG to the DC sub-MG at all times. This applies to this base case and also to the following cases studied, as expected for the considered hybrid MG, where the weaker DC sub-MG calls for support from the AC DERs.

It is important to highlight that the representation of the battery's status in terms of hourly "available capacity" cannot describe the decay trend in the battery, which depends on the number of charges and discharges during each hour. In the real practice, the trend of this decay can be monitored by dedicated EM system apparatuses, comparing the frequency of the charge/discharge to the nominal decay data, which is also important to define the base cost coefficient $\left(c_{B}\right)$ (ref. paragraph $g$ ) of Section III).

\section{b) Case B}

The loss of $50 \%$ of controllable generation capacity causes significant curtailments of controllable AC and DC loads, as well as of EVs, at all times. This is shown in Fig. 4, where the results of Case B are compared with those of Case A. From this figure, it can be seen that the curtailment in Case B is particularly higher during daytime for controllable AC and DC loads, and about $50 \%$ of the initial scheduling at all times for the EVs. This outcome demonstrates the importance of energy demand management. Absence of this functionality in the EM scheme, or limited controllability of the responsive loads, strongly decreases the flexibility of the hybrid MG operation and the reliability of power supply to customers.

Simulation results also show that the loss of a controllable generation unit impacts on the patterns of interlinking converter's power conversion from AC sub-MG to DC sub-MG, as well as of battery dispatch. This is clearly represented in Fig. 5's subplots: compared to Case A, the interlinking converter's power transfer is generally reduced over the 24 hours (up to $50 \%$ ), and the free storage space within the battery is significantly lower during morning (09:00-13:00) and in the early evening (18:00-24:00). Such patterns highlight that the AC/DC power conversion is generally exploited for electricity dispatch over the hours, evidently compensating the possibly reduced supply potential of the controllable generation, alongside the wind power source and the battery storage.

\section{c) Case C}

Constraints (12)-(14) are relaxed to assess the sensitivity of controllable loads and EVs to the absence of battery storage in the AC sub-MG.

Results in Fig. 6 compare this case with the base Case A, showing that the exclusion of the battery from the EM (Case C) yields a significantly higher curtailment of controllable AC loads and EVs, particularly during daytime. The curtailment of controllable DC loads even reach the unacceptable level of $100 \%$ of the scheduling at some hours. Furthermore, the controllable generation is forced to work closer to its limits.

The outcomes of this case can be detrimental not only to the hybrid MG security (i.e., the MG components - in this case the controllable generation - are forced to work in more stressful conditions), but also to the electricity users' satisfaction (due to the dramatically increased curtailment needs). This indicates the importance of embedding battery storage support in the EM scheme, and reinforces the findings of Case B about the benefits of joint energy demand management integration. 


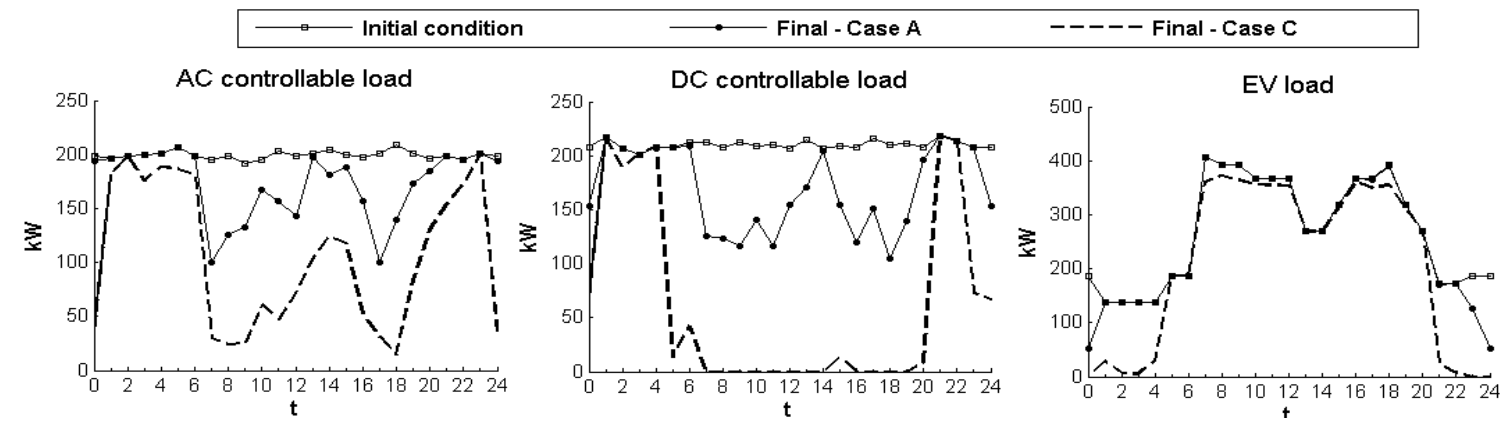

Fig. 6. Operating conditions of interlinking converter and AC and DC controllable loads in the absence of battery energy storage (Case C)

\section{d) Case D}

A parametric analysis is carried out by reducing the interlinking converter rating, to assess the relationship between the power transfer capability of the device and the operating behavior of the other resources within the MG. Comparing the results of this analysis with those of Case A, some conclusions can be drawn on the optimal size of the converter.

Specifically, results show that reductions of the interlinking converter capability up to $500 \mathrm{kVA}$ do not affect the other resources' operating pattern, but further reductions yield infeasibility of the optimal scheduling problem. This suggests that, a 500kVA-sized interlinking converter can be optimal (all other conditions being equal to those assumed in Case A), not only for the hybrid MG operation, but also for saving on initial investment costs and future maintenance costs related to the presence of the converter device.

\section{e) Case E}

A parametric analysis is carried out to assess the impact of EV controllability reduction on MG's operation and costs. The controllability is progressively decreased by $5 \%$ steps of reduction, from $85 \%$ up to reaching infeasibility (at about $45 \%$ ).

The simulations show that the reduced controllability of EVs does not impact on the hybrid MG operation in global terms, as it only affects - but not significantly with respect to Case A the curtailment of controllable AC and DC loads in certain hours of the daytime. Correspondingly, some economic impact can be seen, as the total costs of operating the MG over the 24hours slightly raise with respect to the base case (ref. Fig. 12).

\section{f) Case F}

To prove further the flexibility of the ED model, its sensitivity to the energy service parameters is investigated, considering two aspects. The first is the possible range of energy users' requirements, and the second is the influence of a time-based costing of the MG operation. The latter is an important parameter to consider in the operation of the available resources over the day.

The hybrid MG operating conditions are assessed with a different ratio of renewable and controllable resources (solar and controllable generation, battery storage, and controllable loads including EVs) to critical demand, and considering a timebased tariff set for the controllable generators' dispatch. Then, the initial assumptions, discussed in sub-section IV.A for Case
A, are modified as follows:

- Critical AC and DC loads are deterministically remodeled as residential profiles with typical morning and evening peak demands. These profiles are shown in Fig. 7, alongside the other load profiles ("mean value" profiles for the stochastic controllable loads).

- Contracted bands of energy demand management on controllable loads and EVs are assumed equal to $100 \%$, to assess the sensitivity of the energy demand management actions to the considered scenario.

- The solar power production is remodeled as dependent on the light conditions (solar radiation), according to the daily output profile of a PV unit not supported by storage or additional generators. The mean values, over all probabilistic scenarios, of the selected profile is depicted in Fig. 8.

- The unit cost of controllable generators' power supply is remodeled on a time-of-use basis, assuming: $\rho_{C G U}=0.08 € l$ $\mathrm{kWh}$ at 00:00-07:59, $\rho_{C G U}=0.12 € / \mathrm{kWh}$ at 08:00-17:59, and $\rho_{C G U}=0.16 € / \mathrm{kWh}$ at 18:00-23:59. These values are referred to historical pricing data of European electricity tariffs.

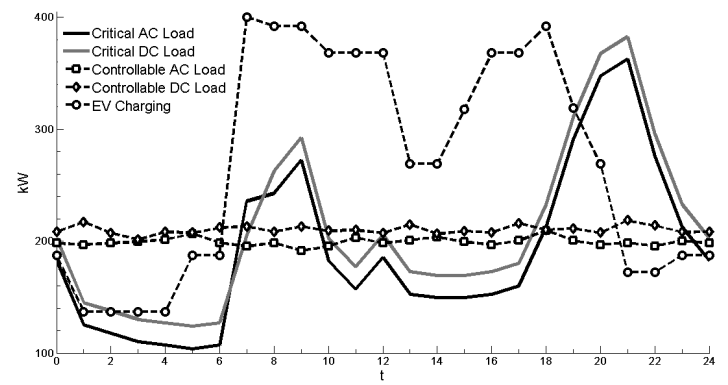

Fig. 7. Hourly load demand in Case F

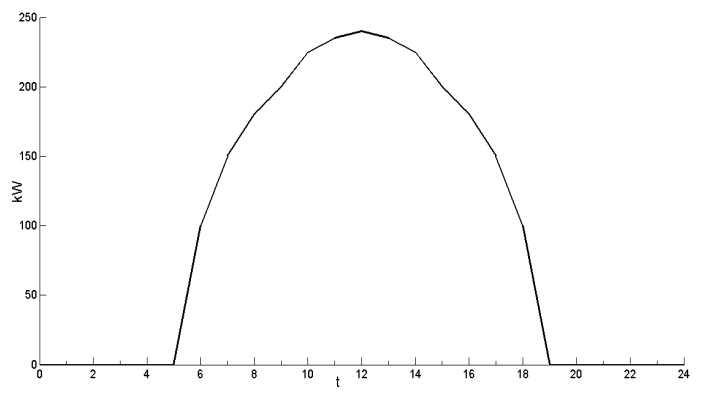

Fig. 8. Purely solar power production in Case F (mean values over probabilistic scenarios) 


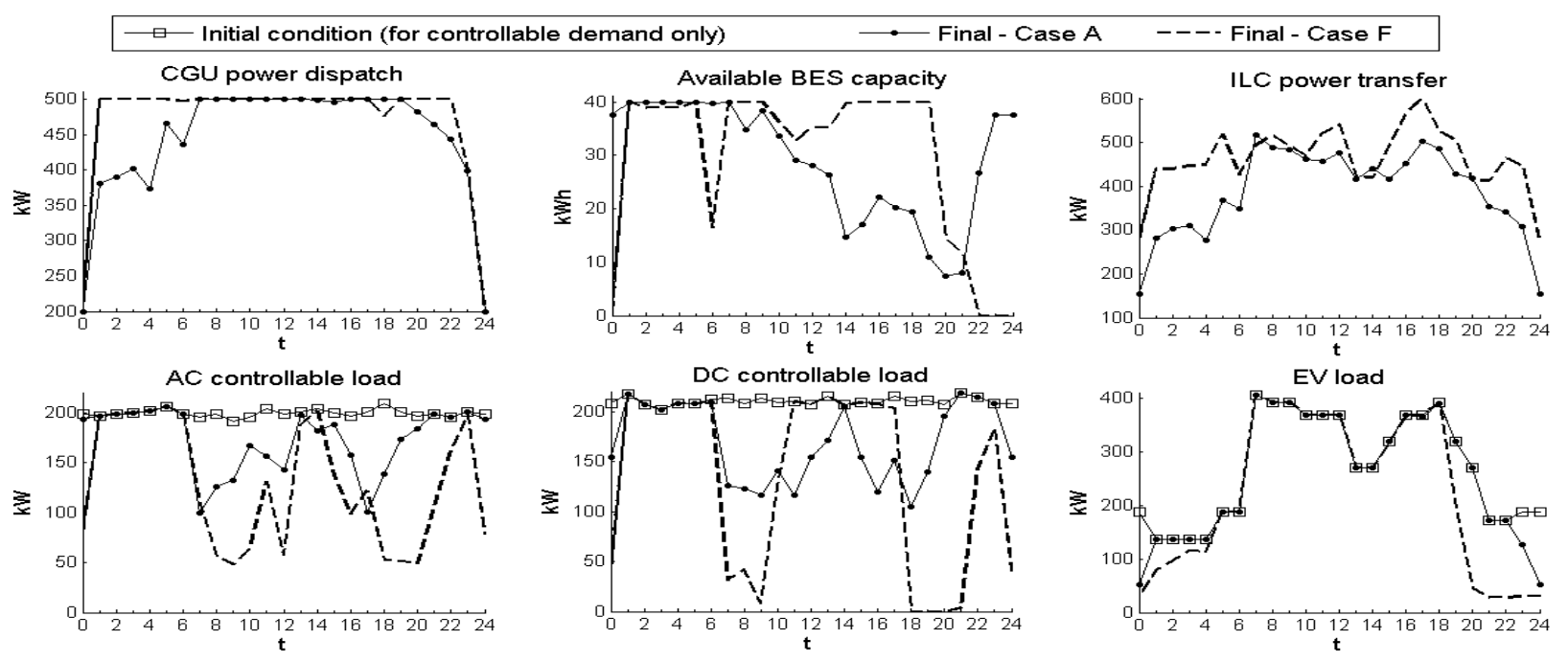

Fig. 9. Comparison of optimal operating conditions of the hybrid MG resources in Case A and Case F

Fig. 9 shows the results of this case compared to those obtained in Case A, where a fixed tariff was assumed for the controllable generators to investigate the MG operation from a mere technical perspective not influenced by timed-based pricing strategies of electricity providers. In this figure, the "CGU dispatch" and "available BES capacity" subplots show that the controllable generation and the battery work coordinately with the RESs, dispatching electricity to meet the critical load needs when the solar power is not available. The fact that the controllable generation works at full capacity, demonstrates that its operating condition is influenced more by the availability of the other resources than by the time-based pricing.

As for the controllable AC and DC loads, Fig. 9 shows that these are subject to more curtailment than in Case A during daytime, and especially during the hours of critical peak demand (07:00-12:00 and 18:00-23:00). Instead, the EV charging is generally curtailed during evening and early morning (when the demand management price is low), more in the hours of evening peak demand (19:00-24:00). So, the energy demand management actions compensate the peak demand from critical loads during low light conditions as well as the higher cost of electricity supply from controllable generators.

Finally, the interlinking converter transfers power to the DC sub-MG according to the load balance requirements, at higher rates during the peak demand hours.

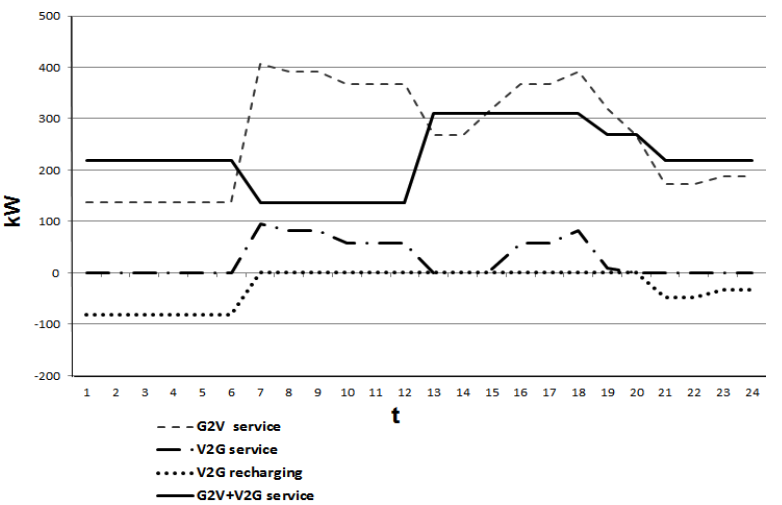

Fig. 10. Leveling of EV load following V2G-based program (Case G)

\section{g) Case G}

Compared to Case F, Case $\mathrm{G}$ introduces the V2G service into the ED simulation records, assuming that the EV charging station is provided with an extra battery stock correspondent to a predetermined amount of energy reserved for V2G delivery. The battery stock is detracted from a mix of recharged swapping and onboard battery packages available as storage resources in the EV charging station. The considered scenario is presented in Fig. 10: a daily V2G program is carried out from 07:00 to 20:00, to level the EV absorption profile, and the used
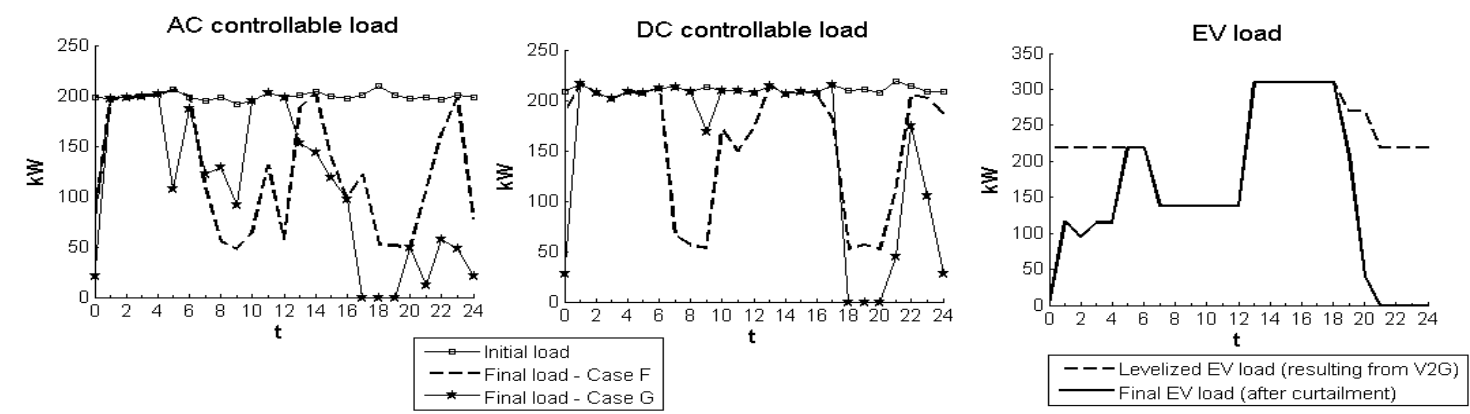

Fig. 11. Load management actions in Case G 
batteries are recharged overnight within the low cost time slot (so that the EV service agent can reduce the recharging costs).

The difference between the unitary costs of bidirectional $\mathrm{V} 2 \mathrm{G}$ service in case $\mathrm{G}$ and of unidirectional $\mathrm{EV}$ service in case $\mathrm{F}$ is considered not significant.

Fig. 11 depicts some relevant outcomes of Case G. In this figure, the results of energy demand management on $\mathrm{AC}$ and DC loads in Case G and Case F are compared (ref. first and second subplots), and the final EV charging load (after the V2G leveling and the following load management action) is shown. Following the V2G-based leveling of EV load at the DC bus, as represented in Fig. 11, the curtailment of both AC and DC controllable loads is generally reduced over the daytime, compared to case F. Specifically, the scheduling of the DC controllable loads appears unaltered during the daytime, and curtailed only in a few evening hours. So, the V2G service is particularly beneficial for the DC sub-microgrid in reducing the load sharing margins of the internal resources, contributing to the overall improvement of the load management in the hybrid MG. The EV service operator can use V2G to manage the contracted bands of EV load sharing and shedding, based on the economic objective of the hybrid MG.

\section{h) Comments on optimal operating costs and resources contri- bution}

The optimal values of the objective function, which are given in Fig. 12 for all simulated cases, indicate a clear alignment between the economic results and the technical outcomes. In fact, higher hybrid MG operation's costs can be seen in the most inconvenient operating scenarios, i.e., when the controllable generation's capacity is reduced (Case B, $€ 14,933$ ), or when the battery energy storage is not included in the EM (Case C, $€ 18,637$ ), or when the EVs have reduced controllability (Case $\mathrm{E}, € 9,486)$. On the contrary, the most cost-effective operation is realized in the other cases (Case A, $€ 9,475$ and Case D, $€ 9,395)$, when all resources are available and managed according to the base EM scheme.

Case D (500kVA-sized interlinking converter) yields the most economic operating costs ( $€ 9,395)$. This reinforces the findings discussed in the previous sub-section for Case $\mathrm{D}$, indicating that, for the hybrid MG selected, and under all other assumptions of Case A, the reduced size of interlinking converter is optimal, because it does not affect the operational performances of the MG resources and has yet the best level of costeffectiveness.

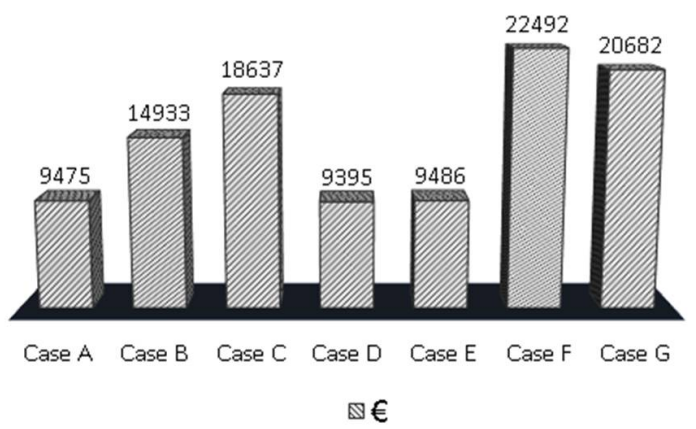

Fig. 12. Total costs of the hybrid microgrid operation in the different cases

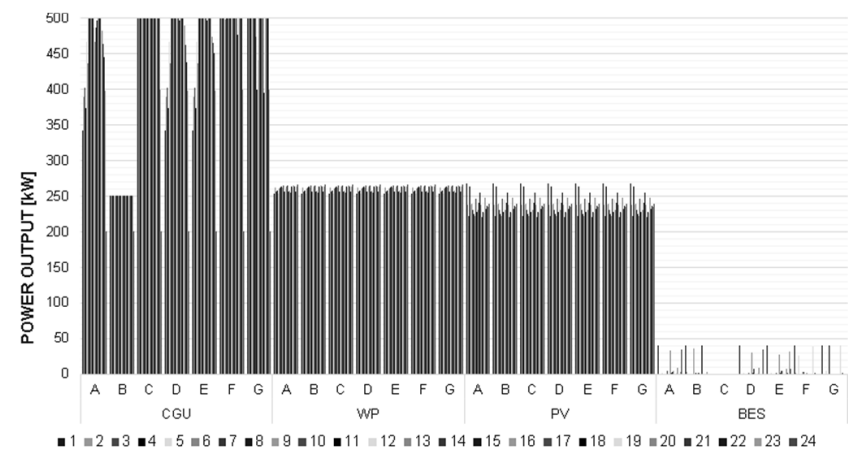

Fig. 13. Contribution to power dispatch of generating and storage resources

The highest costs of the MG operation in Case F ( $€ 22,492)$ reflect the dynamics of the time-based pricing strategy actuated on the different services by the respective sellers (being them the resources' owners or the MG operator). In this case, the time-of-use pricing better represents the true cost of power. It charges more the end-users when the electricity is more expensive to produce, and encourages to use power when rates are lowest. Compared to Case F, the lower costs obtained in case $\mathrm{G}$ ( $€ 20,682)$ indicate the economic benefit of introducing load leveling by V2G into the EM program of the DC sub-MG.

Fig. 13 shows the participation in the power dispatch of generation and battery storage, over the 24 hours and in all cases considered. In this figure, it can be seen that the potential of both controllable generation and RESs is fully utilized at most of the times, so that the battery storage discharges power just according to the unsatisfied balance requirements and to the objectives of minimizing its costs and degradation.

\section{CONCLUSIONS}

This paper has proposed an EM approach, supported by an ED tool, for the efficient and reliable operation of remote hybrid AC/DC MGs. The approach is robust to possible uncertainties, and accordingly the modeling considers the probabilistic behavior of certain parameters related to the available energy service. The EM approach relies on battery energy storage and energy demand management.

Studies on a test hybrid MG have validated the proposed EM scheme and models in a variety of operating scenarios. One of these scenarios considers the use of V2G for energy efficiency purposes, in particular load leveling at the DC bus, which improves the total power absorption profile of the hybrid MG. The results have proved the effectiveness of the ED tool and its practicality and flexibility in operation under different conditions, The implementation of the methodology into the case study has highlighted that the ED tool manages a variety of aspects in hybrid MGs, such as the optimal sizing of the interlinking converter.

The ED tool can support planning studies, serving as a simulator. Its effectiveness, flexibility and practicality in operation make it even more suitable for the scheduling of the hybrid MG, with the integration of procedures for the data updating.

The probabilistic structure of the models allows a scalable 
assessment of uncertainties and challenges in hybrid MGs' design. In this respect, the applications presented in this work have shown the advantages of integrating battery storage and energy demand management in the EM system, in terms of reliability and cost-effectiveness of power supply.

With the modeling of the cost of battery participation in the dispatch service, this work gives some methodological cues about the practical and applicative characterization of the battery decay, which is particularly critical to assign an economic value to the service of the device. This challenge is conceptually raised in this work, and will be the specific subject of future studies.

\section{REFERENCES}

[1] N. Hatziargyriou, Microgrids: Architectures and Control. Wiley-IEEE Press. Feb 2014

[2] M. E. Baran and N. R. Mahajan, "DC distribution for industrial systems: Opportunities and challenges," IEEE Trans. Ind. Appl., vol. 39, no. 6, pp. 1596-1601, Nov. 2003.

[3] D. Salomonsson and A. Sannino, "Low-voltage de distribution system for commercial power systems with sensitive electronic loads," IEEE Trans. Power Del., vol. 22, no. 3, pp. 1620-1627, July 2007.

[4] D. J. Hammerstrom, "AC versus DC distribution systems: Did we get it right?," in Proc. 2007 IEEE-PES General Meeting, pp. 1-5, 24-28 June 2007.

[5] Peng Wang, L. Goel, Xiong Liu, Fook Hoong Choo, "Harmonizing AC and DC: A Hybrid AC/DC Future Grid Solution," IEEE Power Energy Mag., vol.11, no.3, pp.76-83, May-June 2013.

[6] J.M. Guerrero, Poh Chiang Loh, Tzung-Lin Lee, M. Chandorkar, "Advanced Control Architectures for Intelligent Microgrids-Part II: Power Quality, Energy Storage, and AC/DC Microgrids," IEEE Trans. Ind. Electron., vol.60, no.4, pp.1263-1270, April 2013.

[7] Liu Xiong, Peng Wang, and P. Loh, "A hybrid AC/DC microgrid and its coordination control," IEEE Trans. Smart Grid, vol. 2, no. 2, pp. 278286, Jun. 2011

[8] P. Loh, D. Li, Y. Chai, and F. Blaabjerg, "Autonomous operation of hybrid microgrid with AC and DC sub-grids," IEEE Trans. Power Electron., vol. 28, no. 5, pp. 2214-2223, May 2013

[9] Xiaonan Lu, J.M. Guerrero, Kai Sun, J.C. Vasquez, R. Teodorescu, and Lipei Huang, "Hierarchical Control of Parallel AC-DC Converter Interfaces for Hybrid Microgrids," IEEE Trans. Smart Grid, vol.5, no.2, pp.683,692, March 2014.

[10] A. A. A. Radwan and Y. A. Mohamed, "Assessment and mitigation of interaction dynamics in hybrid AC/DC distribution generation systems," IEEE Trans. Smart Grid, vol. 3, no. 3, pp. 1382-1393, Sep. 2012.

[11] J. Elizondo, J.L. Kirtley, "Effect of inverter-based DG penetration and control in hybrid microgrid dynamics and stability," in Proc. 2014 Power and Energy Conf. at Illinois, pp.1-6, Feb. 28 - March 1, 2014

[12] M. Farhadi, O. Mohammed, "Real time Operation and Harmonic Analysis of Isolated and Non-Isolated Hybrid DC Microgrid," IEEE Trans. Ind. Appl., no.99, Jan. 2014.

[13] C. Tsung, Po Chen, "Zero-sequence circulating current reduction method for parallel HEPWM inverters between AC bus and DC bus," IEEE Trans. Ind. Electron., vol. 59, no. 1, pp. 290-300, Jan. 2012.

[14] C. Wang, X. Li, L. Guo, Y. Li, "A Nonlinear-Disturbance-ObserverBased DC-Bus Voltage Control for a Hybrid AC/DC Microgrid," IEEE Trans. Pow. Electron, vol.29, no.11, pp.6162-6177, Nov. 2014.

[15] R. Majumder, "A Hybrid Microgrid with DC Connection at Back to Back Converters," IEEE Trans. Smart Grid, vol.5, no.1, pp.251-259, Jan. 2014.

[16] C. Battistelli, L. Baringo, A.J. Conejo, Optimal energy management of small electric energy systems including V2G facilities and renewable energy sources, Elect. Power Syst. Res., vol.92, pp. 50-59, Nov. 2012.

[17] Quanyuan Jiang, Meidong Xue, Guangchao Geng, "Energy Management of Microgrid in Grid-Connected and Stand-Alone Modes," IEEE Trans. Power Syst., vol.28, no.3, pp.3380-3389, Aug. 2013.

[18] M. Mao, P. Jin, N.D. Hatziargyriou, L. Chang, "Multiagent-Based Hybrid Energy Management System for Microgrids," IEEE Trans. Sustain. Energy, vol.5, no.3, pp.938-946, July 2014.
[19] D.E. Olivares, C.A. Canizares, M. Kazerani, "A Centralized Energy Management System for Isolated Microgrids," IEEE Trans. Smart Grid, vol.5, no.4, pp.1864-1875, July 2014.

[20] Inderjeet Duggal, "AC-DC Microgrid Optimal Power Flow", B.Eng. Thesis, Ryerson University, Toronto, ON, Canada, 2012.

[21] C. Battistelli, "Generalized microgrid-to-smart grid interface models for vehicle-to-grid," in Proc. 2013 IEEE-PES Conf. on Innovative Smart Grid Technol., pp.1-6, 24-27 Feb. 2013.

[22] C. Battistelli and A.J. Conejo, "Optimal management of the automatic generation control service in smart user grids including electric vehicles and distributed resources," Elect. Power Syst. Res., vol. 111, pp. 22-31, June 2014.

[23] A. Rajendra Prasad, E. Natarajan, "Optimization of integrated photovoltaic-wind power generation systems with battery storage," Energy, vol. 31, no. 12, pp. 1943-1954, Sep. 2006.

[24] Takuro Sato, Smart Grid Standards: Specifications, Requirements, and Technologies. Wiley, Apr 2015.

[25] A. Whitman, et al., "Electric Vehicle Grid Integration in the U.S., Europe, and China," Report, July 2013.

[26] S. Shinzaki, H. Sadano, Y. Maruyama, W. Kempton, "Deployment of Ve hicle-to-Grid Technology and Related Issues," SAE Technical Paper 2015-01-0306, 2015.

[27] Sanjoy Kumar Nandi, Himangshu Ranjan Ghosh, "A wind-PV-battery hybrid power system at Sitakunda in Bangladesh”, Energy Policy, vol. 37, no. 9, Sep 2009.

[28] J. Li, W. Wei, J. Xiang, "A Simple Sizing Algorithm for Stand-Alone PV/Wind/Battery Hybrid Microgrids," Energies, vol. 5, no. 12, pp. 53075323, Dec 2012.

[29] S. Sarabi, A. Bouallaga, A. Davigny, B. Robyns, V. Courtecuisse, Y. Riffonneau, M. Régner, "The Feasibility of the Ancillary Services for Vehicle-to-Grid Technology", in Proc. 11th Intl. Conf. on the European Energy Market (EEM14), pp.1-5, May 282014

[30] F. Bouffard and F. D. Galiana, "Stochastic security for operations planning with significant wind power generation," IEEE Trans. Power Syst., vol. 23, no. 2, pp. 306-316, May 2008.

[31] J.M. Morales, R. Mínguez, A.J. Conejo, "A methodology to generate statistically dependent wind speed scenarios," Applied Energy, vol. 87, no. 3, pp. 843-855, March 2010.

[32] J. Dupacová, N. GröweKuska, and W. Römisch, "Scenario reduction in stochastic programming: An approach using probability metrics," Math. Programm., vol. 95, pp. 493-511, 2003.

[33] C. Battistelli, M. Uccelletti, "Assessing wind power and electrical power systems interconnection: a methodological approach," in Proc. IEEE Power Tech, pp. 1-7, 19-23 June 2011.

[34] W. Charytoniuk, M.S. Chen, P. Kotas, and P. Van Olinda, "Demand forecasting in power distribution system using nonparametric probability density estimation," IEEE Trans. Power System, vol. 14, pp. 1200-1206, Nov. 1999.

[35] X. P. Zhang, C. Rehtanz, and B. Pal, Flexible ac Transmission Systems, Modelling and Control. Berlin, Germany: Springer 2006.

[36] X. Wei, J. H. Chow, B. Fardanesh, and A. Edris, "A common modeling framework of voltage-sourced converters for power flow, sensitivity, and dispatch analysis," IEEE Trans. Pow. Sys., vol. 19, no. 2, pp. 934-941, May 2004.

[37] M.D. Galus, S. Koch, G. Andersson, Provision of load frequency control by PHEV s, controllable loads, and a cogeneration unit, IEEE Trans. Ind. Electron., vol. 58, no. 10, pp. 4568-4582, Oct 2011.

[38] V.D.N. Santos, J.P. Trovao, T.P. Branco, J.M.R. Goncalves, "Information and Communication Technology Solution for the V2G Concept Implementation," in Proc. IEEE Vehicle Power and Propulsion Conf. (VPPC 2014), pp.1-6, Oct 27, 2014.

[39] M. Koller, T. Borsche, A. Ulbig, G. Andersson, "Defining a degradation cost function for optimal control of a battery energy storage system," in Proc. of 2013 IEEE PowerTech, pp.1-6, 16-20 June 2013.

[40] Energy Modeling Software for Hybrid Renewable Energy System. HOMER Energy: Boulder, CO, USA. Available online: http://www.homerenergy.com/download.html.

[41] Jizhong Zhu, Optimization of Power System Operation (2nd Edition). Wiley-IEEE Press, Feb 2015

[42] Lo, Y.K. Wu, "Analysis of relationships between hourly electricity price and load in deregulated real-time power markets," IEE Proc. Gen. Transm. Distrib., vol. 151, pp. 1350-2360, July 2004. 
Claudia Battistelli (M'08) received her MS (2006) and PhD (2010) degrees in Electrical Engineering from the Federico II University of Naples, Italy. She is currently a Research Associate at the Electrical and Electronic Engineering Department of Imperial College, London, UK. Her research interests are in the fields of power systems planning, operations and economics, smart grids, decision-making under uncertainty, and electricity markets.

Yashodhan P. Agalgaonkar (M'14) received an M.Sc. in Electrical Power Engineering from the Chalmers University of Technology, Gothenburg, Sweden, in 2006, and a Ph.D. in Electrical Power Engineering from Imperial College London, London, U.K., in 2014. He was a Postdoctoral Researcher at Imperial College London until 2014. From 2006 to 2010, he was with Crompton Greaves, India and with Converteam (now GE Energy), Chennai, India and Berlin, Germany, as a Research Engineer. During his tenure at Crompton Greaves and Converteam, he conducted research on diverse areas of power transmission and distribution operation. This work was carried out during the period the Imperial College London, London, U.K. Since 2015 he has been a Scientist and Engineer in the Energy and Environment Division of Pacific Northwest National Laboratory, Richland, Washington.

Bikash C. Pal (M'00-SM'02-F'13) received the B.E.E. (with honors) degree from Jadavpur University, Calcutta, India, the M.E. degree from the Indian Institute of Science, Bangalore, India, and the Ph.D. degree from Imperial College London, London, U.K., in 1990, 1992, and 1999, respectively, all in electrical engineering. Currently, he is a Professor in the Department of Electrical and Electronic Engineering, Imperial College London. His current research interests include state estimation, and power system dynamics. Prof. Pal is Editor-inChief of the IEEE TRANSACTIONS ON SUSTAINABLE ENERGY and Fellow of IEEE for his contribution to power system stability and control. 